\title{
Vildagliptin - Mode of Action
}

\section{James Edward Foley*}

Director Global Medical Affairs, Novartis Pharmaceuticals Corporation, 100 West Village Promenade, East Hanover, NJ 07936-1080, USA

Incretin hormones, glucagon-like peptide 1 (GLP-1) and glucose dependent insulinotropic polypeptide (GIP) are important for normal pancreatic islet function and glucose homeostasis. There is a surge in GLP-1 and GIP secretion at the beginning of each meal followed within minutes by their rapid inactivation by the enzyme dipeptidyl peptidase 4 (DPP-4). Both GLP-1 and GIP increase the sensitivity to glucose of the $\beta$-cells and thus enhances glucose-dependent insulin secretion, GLP-1 also improves the sensitivity to glucose of the $\alpha$-cells under hyperglycemic conditions and thus reduces inappropriate glucagon secretion thereby decreasing insulin resistance [1], and GIP improves the sensitivity of the $a$-cell to glucose under hypoglycemic conditions and thus enhances glucagon counter-regulation [2].

Independent of GLP-1 and GIP action, the sensitivities to glucose of the $\alpha$ and $\beta$-cells of the pancreas are diminished in type 2 diabetes (T2DM) leading to impaired insulin secretion, insulin resistance due to elevated glucagon levels in hyperglycemia and impaired glucagon counter regulation in hypoglycemia[1]. In addition, T2DM is associated with increased lipo-toxicity induced insulin resistance due inappropriate fasting lipolysis leading increased stored triglyceride in liver, muscle and $\beta$-cells [3-4].

Vildagliptin (LAF237), a member of the DPP-4 inhibitor class of oral antidiabetic drugs for the treatment of T2DM, prolongs the meal induced increases of GLP-1 and GIP by blocking the enzyme DPP-4, leading to improved glucose control1. In general DPP-4 inhibitors prolong the physiological meal induced increase in GLP-1 and GIP. How long these levels are prolonged depends on how complete the DPP-4 inhibition is. A 50\% inhibition would extend the half-life 2-fold, a $90 \%$ inhibition 10 -fold, a $95 \%$ inhibition 20 -fold and with $100 \%$ inhibition the half-life would be determined by the renal clearance of GLP-1 and GIP. Competitive DPP-4 inhibitors, such as sitagliptin, which achieve 90-95\% inhibition of DPP-4 can maintain GLP-1 and GIP levels above the threshold required for their actions in the pancreas over the course of each meal, falling below this threshold between meals and overnight. Vildagliptin is a slow substrate for DPP-4 which blocks GLP-1 and GIP inactivation over 24 hours resulting in maintenance of GLP-1 and GIP levels above the threshold for their actions in the pancreas over the entire 24 hours of each day.

Most DPP-4 inhibitors are competitive inhibitors that follow simple Michaelis-Menton kinetics and dose dependently inhibits the enzyme. For example, if a given dose produces $90 \%$ inhibition then it will require a 10 -fold higher dose to achieve $99 \%$ inhibition. Vildagliptin has a nitrile group which rapidly forms a covalent bond with the catalytic site of DPP-4 to form an imidate group, which stabilizes vildagliptin in the catalytic site of DPP-4 and facilitates hydrolysis of this imidate group. Inactive vildagliptin then slowly dissociates from the catalytic site with a half-life of about $1 \mathrm{~h}$. While vildagliptin is covalently bound to the DPP-4 catalytic site, the enzyme cannot act on any other substrate. Following dissociation of vildagliptin from the catalytic side, within a fraction of a second, another vildagliptin molecule will interact with the catalytic site. This leads to complete blocking of DPP-4 activity over the entire time that vildagliptin drug levels are adequate to effectively associate with the catalytic site; a vildagliptin drug level of $50 \mathrm{nM}$ is adequate to maintain this block. Despite a half-life of $\sim 2$ hours, $50 \mathrm{nM}$ is remaining for at least 10 hours after a $50 \mathrm{mg}$ dose of vildagliptin [1].

In clinical studies performed on the effect of vildagliptin on $\alpha$-cell sensitivity, it is shown that vildagliptin improves $\alpha$-cell sensitivity to glucose in the hyperglycemic range of glucose which reduces the glucagon levels in hyperglycemia. Vildagliptin has also been shown to improve the $\beta$-cell sensitivity to glucose leading to increased insulin secretion when glucose levels are above normal. As a result of improved $\alpha$ - and $\beta$-cell responsiveness to glucose, the insulin to glucagon ratio increases in hyperglycemia, thereby reducing the rate of hepatic glucose production and lowering blood glucose levels. In hypoglycaemia, the insulin to glucagon ratio decreases promoting the rate of hepatic glucose production which prevents hypoglycemial. In non-diabetic (normoglycaemic) individuals, vildagliptin does not stimulate insulin secretion, does not reduce glucagon levels and does not reduce glucose levels [5].

When patients with T2DM have been treated with insulin and glucose levels are further reduced by the addition of an oral agent, the hypoglycemia rate is expected to increase in the face of insulin therapy at a lower level of glycemic control. Vildagliptin lowers glucose levels without increasing hypoglycemia when added to insulin therapy [2]. Thus, vildagliptin appears to confer protection against hypoglycemia not seen with other oral agents. When patients are treated with insulin, the first line of defense when glucose levels fall below normal is increased glucagon secretion by the a-cells of the pancreas. GIP increases the sensitivity of the a-cells to glucose in the hypoglycemic range of glucose levels thereby enhancing glucagon secretion in response to hypoglycemia which in turn would confer protection against hypoglycemia [2]. Since hypoglycemia is most evident between meals and overnight, elevations in GIP during this period would be necessary in order to confer this protection against hypoglycemia.

Lipotoxicity has been implicated in $\beta$-cell failure and insulin resistance in T2DM3-4. Thiazolidinediones inhibit fasting lipolysis resulting in the redistribution fat out of muscle/liver and $\beta$-cells into adipocytes, leading to enhanced insulin sensitivity and improved $\beta$-cell function via reduced lipotoxicity [3-4]. Vildagliptin also inhibits fasting lipolysis leading to enhanced insulin sensitivity via reduced lipotoxicityl. The REDIRECT (TRiglycEride accumulation anD Insulin REsistanCe assessment on vildaglipTin) [linicalTrials.gov: NCT01356381] Study is being carried out to demonstrate that the vildagliptin treatment also leads to the redistribution fat out of muscle/ liver and $\beta$-cells into adipocytes. Interestingly, in contrast to the fasting state, a post-meal increase in lipolysis in adipose tissue resulting increased lipid oxidation in muscles has also been observed with vildagliptin. Thus, although both thiazolidinediones and presumably vildagliptin collect fat from liver, muscle and $\beta$-cells and store them in adipocytes during the fasting state, adipocyte mass increases with

*Corresponding author: James Edward Foley $\mathrm{PhD}$, Director Global Medical Affairs, Novartis Pharmaceuticals Corporation, 100 West Village Promenade, East Hanover, NJ 07936-1080, USA, Tel: +1 862 7783258, +1 862 6843251, Fax : +1 973 7816619; E-mail: james.foley@novartis.com

Received February 25, 2014; Accepted April 28, 2014; Published April 30, 2014

Citation: Foley JE (2014) Vildagliptin - Mode of Action. Med chem 4: 439-440. doi:10.4172/2161-0444.1000176

Copyright: ( 2014 Foley JE. This is an open-access article distributed under the terms of the Creative Commons Attribution License, which permits unrestricted use, distribution, and reproduction in any medium, provided the original author and source are credited. 
thiazolidinediones but does not do so with vildagliptin; during each meal this fat is mobilized from adipocytes and burned in muscle due to vildagliptin treatment [6].

Vildagliptin also decreases postprandial lipidaemia by the GLP-1 mechanism independent of the pancreatic islets, which presumably results in less postprandial lipid extraction from the gut, which may in turn partially explain the weight neutrality of vildagliptin [1]. Vildagliptin like other DPP-4 inhibitors does not lead to pharmacological effects of GLP-1 to decrease gastric emptying or enhance satiety [1].

In rodent experimental models, vildagliptin improved $\beta$-cell mass by stimulating replication and inhibiting apoptosis of $\beta$-cell turnover. However, these data should be interpreted with caution due to the physiological differences of $\beta$-cell turnover in rodents versus humans. Preliminary results in T2DM patients showed that after 12 weeks of vildagliptin treatment, there was an approximately $20 \%$ increase in maximum acute insulin response (AIRmax) and an approximately $10 \%$ increase in maximum increment in plasma C-peptide (AC-PR max). These findings demonstrate a modest effect of chronic DPP-4 inhibition in increasing the maximal capacity for insulin release in diabetic subjects; this effect was absent in the placebo-treated group. One-year treatment with vildagliptin significantly increased $\beta$-cell secretory capacity. This effect was not maintained after the washout, indicating that this increased capacity was not a disease-modifying effect on $\beta$-cell mass and/or function [7].

\section{References}

1. Ahren B, Schweizer A, Dejager S, et al. (2011) Mechanisms of action of the dipeptidyl peptidase- 4 inhibitor vildagliptin in humans. Diabetes Obes Metab13:775-783.

2. Schweizer A, Foley JE, Kothny W, Ahrén B (2013) Clinical evidence and mechanistic basis for vildagliptin's effect in combination with insulin. Vasc Health Risk Manag 9: 57-64.

3. Taylor R (2013) Type 2 diabetes: etiology and reversibility. Diabetes Care 36 : 1047-1055.

4. Boden G, Cheung P, Mozzoli M, Fried SK (2003) Effect of thiazolidinediones on glucose and fatty acid metabolism in patients with type 2 diabetes. Metabolism 52: 753-759.

5. El-Ouaghlidi A, Rehring E, Holst JJ, et al. (2007) The dipeptidyl peptidase 4 inhibitor vildagliptin does not accentuate glibenclamide-induced hypoglycemia but reduces glucose-induced glucagon-like peptide 1 and gastric inhibitory polypeptide secretion. J Clin Endocrinol Metab 92: 4165-4171

6. Foley JE, Jordan J (2010) Weight neutrality with the DPP-4 inhibitor, vildagliptin: mechanistic basis and clinical experience. Vasc Health Risk Manag 6: 541-548.

7. Foley JE, Bunck MC, Möller-Goede DL, Poelma M, Nijpels G, et al. (2011) Beta cell function following 1 year vildagliptin or placebo treatment and after 12 week washout in drug-naive patients with type 2 diabetes and mild hyperglycaemia: a randomised controlled trial. Diabetologia 54: 1985-1991. 